\title{
Measuring the Accuracy and Precision of the Garmin GPS Positioning in Forested Areas: A Case Study in Taxiarchis-Vrastama University Forest
}

\author{
Vasileios C. Drosos ${ }^{1}$ and Chrisvaladis Malesios ${ }^{2}$ \\ 1. Laboratory of Forest Engineering Sciences and Surveying, Department of Forestry and Management of the Environment and \\ Natural Resources, Democritus University of Thrace, Orestiada 68200, Greece \\ 2. Laboratory of Agricultural Economics, School of Agricultural Development, Democritus University of Thrace, Orestiada 68200 , \\ Greece
}

Received: August 8, 2011 / Accepted: October 20, 2011 / Published: April 20, 2012.

\begin{abstract}
In this paper, it is attempted to examine and compare the performance of two (global positioning system) receivers of different orientation, one recreational and another more precise, in forested areas. In doing this, a field test on horizontal and vertical positional errors of GPS positioning at different points in the forested area of Taxiarchis-Vrastama University forest was conducted. The two GPS receivers were used to determine the positional accuracy of a selected number of points under tree canopies. Specifically, the precision and accuracy of Garmin's GPS positioning at different points were calculated and compared with the corresponding positioning and accuracy of another GPS system, namely the TOPCON GPS. By the calculation of various measures of accuracy and precision suitable for GPS receivers and the use of statistical methods, accuracy between the different receivers differed significantly is shown. Also, regression analysis revealed that the basal area and the number of available satellites are the most important factors for predicting position error.
\end{abstract}

Key words: Accuracy, GPS, precision, positioning under forest canopy.

\section{Introduction}

GPS (global positioning system) is a satellite-based navigational system designed and operated by the US Department of Defense for military and civilian use.

Besides the standard use of navigation, GPS can be also extremely useful in other tasks, for instance in mapping forested areas, such as streams and forest roads, since that mapping by the utilization of a GPS receiver can significantly reduce positioning errors which are inevitable when measuring with conventional instruments, such as for instance the tape measure. Moreover, GPS is until now the only possible

Corresponding author: Vasileios C. Drosos, assistant professor, Ph.D., main research fields: forest cadastre, forest opening up, forest constructions, photogrammetry, technical drawing and environmental impacts assessments. E-mail: vdrosos@fmenr.duth.gr. option in terms of cost and labor when mapping forests on a large scale [1]. On the other hand, the major problem when utilizing a GPS receiver under forest canopy is that the required satellites signal is often weak or unachievable. It is known that the positioning precision and accuracy under forest canopy are markedly lower than in areas with unobstructed sky conditions because trees attenuate or brake GPS signals. Thus, while under a clear sky the positional errors of a standard GPS may not be larger than a few millimeters, when collecting measurements in forests, the various topographic obstacles tend to reduce significantly the positional accuracy obtained by the GPS. Sigrist and others [2] observe that positional errors can be more than ten times greater under forest canopy than when operating in the open sky. Under this perspective, it is 
of interest to examine how forest canopy affects positional errors obtained from GPS, using different types of receivers and different measurement procedures.

The objective of this study is to clarify the performance of the Garmin GPS in forested areas. For comparison purposes, it calculates accuracy and precision of the TOPCON GPS, and compares the accuracy of both systems. Specifically, the two GPS receivers were used to determine the positional accuracy by collecting field measurements of different points and subsequently it can use statistical analysis to examine the accuracy and reliability of the computed positions.

Accuracy and precision are often used to describe how well is the position acquired by GPS receiver. Accuracy is the degree of closeness of an estimate to its true, but unknown value and the precision is the degree of closeness of observations to their means. There is a series of accuracy and precision measures that have been developed.

The most common measures used in previous works to estimate GPS accuracy and precision are CEP (circular error probable), RMS (root mean square error) and DRMS (distance root mean square error). Sawaguchi and others [3] define CEP as the value which half of the data points fall within a circle of this radius centered on truth and a half lie outside this circle and use CEP to estimate GPS positioning at different forest type, antenna height, and season, and to clarify the relationship between sampling number and the convergence of positioning precision. RMS value indicates that approximately 68 percent of the data points fall within this true distance. Yoshimura and Hasegawa [4] use RMS testing on horizontal and vertical positional errors of GPS positioning at different points in forested areas. DRMS should be expressed clearly whether the accuracy value refers only to horizontal or to both horizontal and vertical and indicates that approximately 95 percent of the data points occur with this distance of truth [5]. It is the method proposed to calculate accuracy in the SPS (standard positioning service) [6]. Dana [5] defines 2DRMS as EPE (estimated positional error) and it is used to compare differences between GPS receivers under forest canopies [7].

There are techniques as DGPS (differential global positioning system) that improve precision and accuracy under tree canopies. Hasegawa and Yoshimura [8] achieved a mean error of a 1 to 30-min observation varied between 0.029-0.226 m (without closed tree canopies) and it was 0.415-0.894 m (with closed tree canopies), using Dual-frequency GPS receivers by carrier phase DGPS static surveying. Sawaguchi and others [3] using DGPS got mean CEP95 $=2.80 \mathrm{~m}$ for deciduous broadleaved trees and $4.99 \mathrm{~m}$ for conifers. Additionally they demonstrated that positioning precision was not noticeably improved if the sampling number was around 10. So DGPS improve GPS positioning in precision, accuracy and efficiency because the observation time is shorter $[9,10]$.

\section{Methods and Data}

\subsection{Study Location and Data Collection}

The experiments were conducted at Taxiarchis-Vrastama University forest that is located in the center of the Chalcidice prefecture, a region of northern Greece, specifically in the south and southwest slopes of Holomon Mountain in latitude of $40^{\circ} 23^{\prime}-40^{\circ} 28^{\prime}$ and longitude of $23^{\circ} 28^{\prime}-23^{\circ} 34^{\prime}$, and with an altitude of $320-1,165$ meters. The vegetation of the area is dominated by deciduous forests and is comprised of vegetation zones depending on the flora composition, the rock layer and soil conditions, the aspect and inclination of the particular area, the ambient temperature and the precipitation. Hence, three zones are distinguished: Quercetalia ilicis, Quercetalia pubescentis and Fragertalia.

Two different low-cost GPS receivers were used in the study. The first GPS receiver was the Garmin GPS (Garmin International Inc., Olathe, KS, USA) while the other receiver was the TOPCON (Topcon Corporation, Hasunuma-cho, Itabashi-ku, Tokyo, Japan). 
The GPS positional errors were measured by using the two receivers while walking under the forest canopy.

The test course consisted of 21 points measured simultaneously for both the Garmin and TOPCON receivers, along with the corresponding real coordinates. Separately, in our disposal there is a total number of 29 measurement points for Garmin, 144 measurement points for TOPCON whereas the corresponding true measurement points are up to 65 . Before starting the field test, the reference coordinate of each positioning point was determined.

The field test to acquire the Garmin GPS and TOPCON GPS observations was conducted during the period between July 2010 and July 2011.

\subsection{Accuracy Measures}

If a GPS receiver displays position coordinates that are different from the "true coordinates" of the antenna position, this is position error ${ }^{1}$. A vast variety of measures have been employed for measuring this error, i.e. the degree of conformance between the estimated or measured position.

As concerns the evaluation of the horizontal positional errors, it can distinguish among these measures the DRMS (distance root mean square), which is defined as Eq. (1):

$$
\text { DRMS }=\sqrt{\sigma_{x}^{2}+\sigma_{y}^{2}}
$$

where, $\sigma_{x}$ and $\sigma_{y}$ denote the standard deviation of the positional error along the $\mathrm{x}$ axis and $\mathrm{y}$ axis, respectively and are calculated by the following expressions (Eqs. (2) and (3)):

$$
\begin{gathered}
\sigma_{x}=\sqrt{\frac{\sum_{i=1}^{n}\left(x_{i}-\bar{x}\right)^{2}}{n-1}} \\
\sigma_{y}=\sqrt{\frac{\sum_{i=1}^{n}\left(y_{i}-\bar{y}\right)^{2}}{n-1}}
\end{gathered}
$$

\footnotetext{
${ }^{1}$ Specifically, for each computed position $i(I=1,2, \ldots, \mathrm{n})$, the positional error, say $D_{i}$ is calculated as the deviation between the satellite obtained coordinate from the GPS receiver and the true reference coordinate.
}

Other horizontal position precision measures include the 2DRMS, which is twice the distance root mean square, and CEP (circular error probability), which is the radius of circle centered at the true position, containing the position estimate with probability of $50 \%$, given by Eq. (4):

$$
\mathrm{CEP}=0.62 \sigma_{\mathrm{y}}+0.56 \sigma_{\mathrm{x}}
$$

The radius of the $95 \%$ is often quoted and the term R95 is used. R95 is CEP with the radius of the 95\% probability circle, calculated by the following expression (Eq. (5)):

$$
\mathrm{R} 95=\mathrm{R}\left(0.62 \sigma_{\mathrm{y}}+0.56 \sigma_{\mathrm{x}}\right)
$$

with $R=2.08$ when $\sigma_{y} / \sigma_{x}=1$. The latter two-dimensional precision measures can be easily extended in the three-dimensional space. Thus, SEP (spherical error probable) applies to combined horizontal and vertical precision, given by (Eq. (6)):

$$
\mathrm{SEP}=0.51\left(\sigma_{\mathrm{x}}^{2}+\sigma_{\mathrm{y}}^{2}+\sigma_{\mathrm{z}}^{2}\right)
$$

Corresponding to the CEP in the two dimensions, the MRSE (mean radial spherical error) is the $3 \mathrm{D}$ analogue of the distance root mean square (Eq. (7)):

$$
\operatorname{MRSE}=\sqrt{\sigma_{\mathrm{x}}^{2}+\sigma_{\mathrm{y}}^{2}+\sigma_{\mathrm{z}}^{2}}
$$

Whereas, the 90\% spherical accuracy standard and the $99 \%$ spherical accuracy standard is given by Eqs. (8) and (9):

$$
\begin{aligned}
& 0.833\left(\sigma_{x}^{2}+\sigma_{y}^{2}+\sigma_{z}^{2}\right) \\
& 1.122\left(\sigma_{x}^{2}+\sigma_{y}^{2}+\sigma_{z}^{2}\right)
\end{aligned}
$$

\section{Results and Discussion}

In Tables 1 and 2, descriptive statistics for the positional errors of the two GPS systems are presented, such as minimum and maximum $D_{i}$, average $D_{i}$ and standard deviation of $D_{i}^{2}$.

As one observes, Garmin GPS exceeds the highest

\footnotetext{
${ }^{2}$ The mean positional error was calculated from Eq. (10): $\overline{\mathrm{D}}=\frac{1}{\mathrm{n}} \sum_{\mathrm{i}=1}^{\mathrm{n}} \mathrm{D}_{\mathrm{i}} \quad$ (10). The standard deviation of the positional errors $D_{i}$ was computed using Eq. (11): $\mathrm{SD}=\sqrt{\frac{1}{\mathrm{n}-1} \sum_{\mathrm{i}=1}^{\mathrm{n}}\left(\mathrm{D}_{\mathrm{i}}-\overline{\mathrm{D}}\right)} \quad$ (11).
} 
positional errors in both the $\mathrm{x}$ - and the $\mathrm{y}$-axis, in comparison to the corresponding positional errors of the TOPCON GPS system. As concerns the z-axis positional errors, it can find that Garmin still has the highest maximum positional error ( 7.55 meters), while the maximum TOPCON z-axis positional error is only 1.03 meters. Accordingly, the average positional errors are constantly significantly higher in the Garmin measurements, with the highest average positional error for the Garmin observed along the x-axis (2.2941 meters) and the lowest along the y-axis (1.1829). Once again, it showes that the corresponding average positional errors for the TOPCON are substantially lower. Fig. 1 presents the error bars with the $95 \%$ Cis (confidence intervals) for the average positional errors regarding receivers.

The high values, as well as the high variance of the errors of Garmin GPS are once again verified graphically by inspecting the plots. On the other hand, the TOPCON receiver is shown to exhibit very low errors that additionally do not vary significantly. This result is also met in other related studies, which in general observe that receivers of high accuracy perform well under unfavorable conditions of heavy forest canopy (see, e.g. Ref. [1]).

To see how the positional errors from the two GPS receivers correlate between the three axes (northing, easting and vertical), Pearson correlation coefficients $^{3}[11]$ have calculated between positional errors of each pair of axes.

Fig. 2 displays the Pearson correlation coefficients along with fitted lines of simple linear regressions between the combinations of the positional errors of the axes. The highest correlations are observed between

\footnotetext{
${ }^{3}$ Pearson's $r$ correlation coefficient, for two continuous variables $\mathrm{X}$ and $\mathrm{Y}$, for which a sample $\mathrm{n}$ is obtained is given by Eq. (12):

$$
r=\frac{\sum_{i=1}^{n}\left(X_{i}-\bar{X}\right)\left(Y_{i}-\bar{Y}\right)}{\sqrt{\sum_{i=1}^{n}\left(X_{i}-\bar{X}\right)^{2} \sum\left(Y_{i}-\bar{Y}\right)^{2}}}
$$

where $\bar{X}$ and $\bar{Y}$ are the corresponding sample means.
}

the $y$ - and $z$-axis positional errors of the Garmin GPS ( $r$ $=0.336$ ), whereas the lowest is between the $\mathrm{x}$ - and $\mathrm{z}$-axis positional errors again for the Garmin. TOPCON on the other hand has shown similar correlation coefficients for all three combinations of axes. From the inspection of the scatter plots and the correlation coefficients' values it is apparent that the vertical positional errors for the Garmin GPS co-vary significantly with the positional errors of the $y$-axis, and exceed minimum co-variation with the $\mathrm{x}$-axis positional errors.

When it comes to the precision of the two systems, the calculated Distance Root Mean Square (DRMS) for Garmin and TOPCON were 2.151787 meters and 0.061368 meters, respectively, indicating thus the lack of precise estimation as concerns the horizontal precision of the Garmin GPS. This result for the horizontal precision of the Garmin GPS is directly comparable with the horizontal precision outcomes in the study of Rodriguez-Pérez and others [12], who also report poor performance of low-cost receivers such as the Garmin GPS. Table 3 shows the different accuracy measures calculated depending on the receiver type.

As we see, the values of the accuracy measures for Garmin GPS are constantly larger when compared to the corresponding values of the TOPCON. For instance, the DRMS for the Garmin is 2.151787, while the horizontal accuracy described by the DRMS for the TOPCON is only 0.061368 . By this, it concludes that with the Garmin receiver, it will fall within 2.151787 meters of the true measurement $65 \%$ of the time, while using the TOPCON they are going to be within 0.061368 meters of the true measurement $65 \%$ of the time, indicating thus that the horizontal accuracy of the Garmin compared with that of the TOPCON is substantially lower. Accordingly, measurements from Garmin with a CEP value of 1.32361 will be within 1.32361 meters of the true measurement $50 \%$ of the time, while the other $50 \%$ of the time the measurements will be in error by more than 1.32361 meters. The corresponding value for the CEP measure for TOPCON is only 0.04887 , which states that by 
Table 1 Descriptive statistics for the positional errors along the $\mathrm{x}, \mathrm{y}$ and $\mathrm{z}$ axes of Garmin GPS.

\begin{tabular}{lllll}
\hline & Minimum $(\mathrm{m})$ & Maximum $(\mathrm{m})$ & Average positional error $(\mathrm{m})$ & Std. Deviation $(\mathrm{m})$ \\
\hline Positional error for Garmin GPS (x axis) & 0.203 & 5.08 & 2.2941 & 1.2451 \\
Positional error for Garmin GPS (y axis) & 0.027 & 3.50 & 1.1829 & 1.0102 \\
Positional error for Garmin GPS (z axis) & 0.0001 & 7.55 & 1.696 & 0.6944 \\
\hline Total & 0.0001 & 7.55 & 1.724 & 1.401 \\
\hline
\end{tabular}

Table 2 Descriptive statistics for the positional errors along the $\mathrm{x}, \mathrm{y}$ and $\mathrm{z}$ axes of TOPCON GPS.

\begin{tabular}{lllll}
\hline & Minimum $(\mathrm{m})$ & Maximum $(\mathrm{m})$ & Average positional error $(\mathrm{m})$ & Std. Deviation $(\mathrm{m})$ \\
\hline Positional error for TOPCON GPS (x axis) & 0.011 & 0.22 & 0.1298 & 0.053 \\
Positional error for TOPCON GPS (y axis) & 0.003 & 0.12 & 0.0566 & 0.03 \\
Positional error for TOPCON GPS (z axis) & 0.011 & 1.03 & 0.1278 & 0.246 \\
\hline Total & 0.003 & 1.03 & 0.105 & 0.148 \\
\hline
\end{tabular}
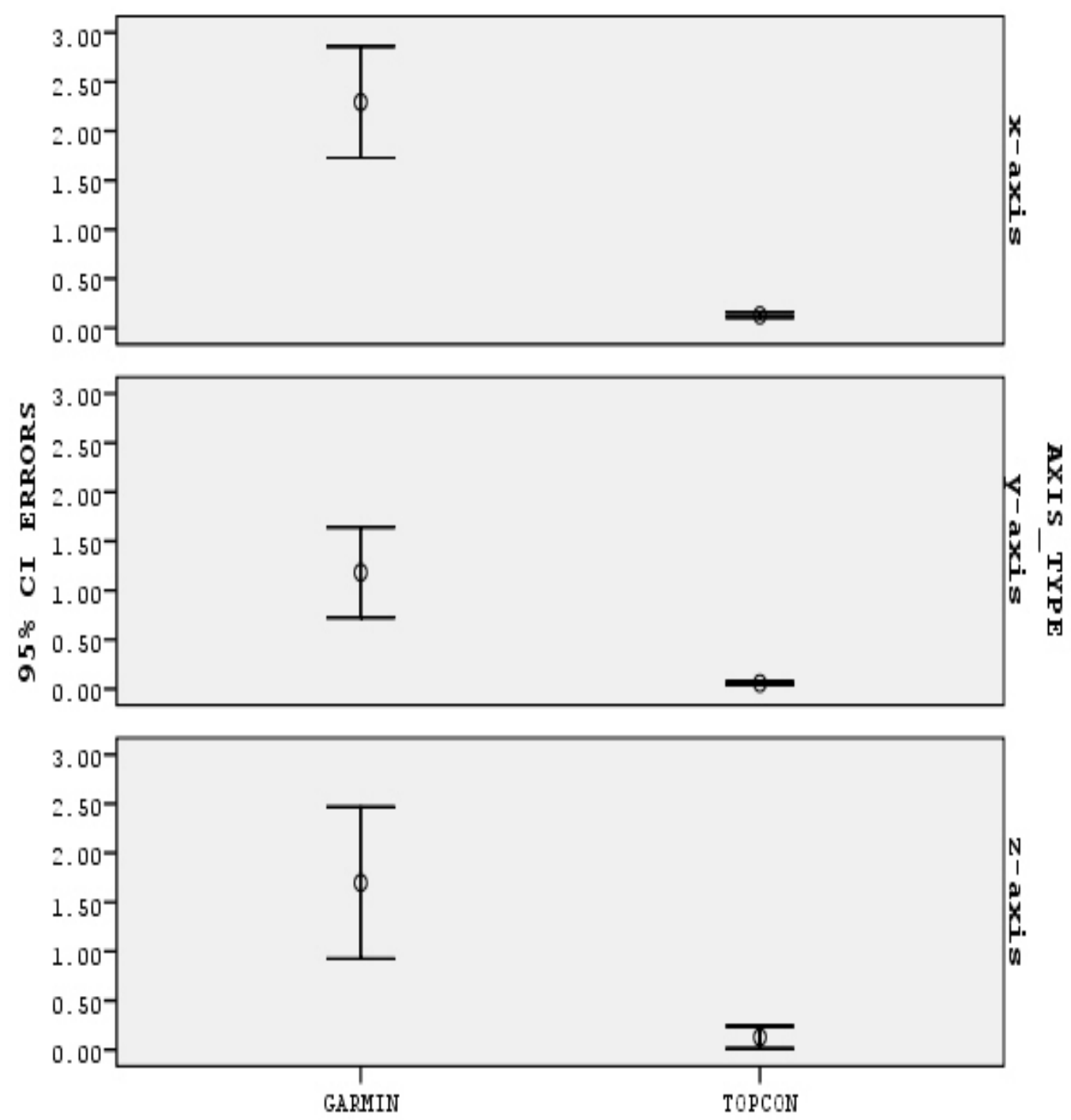

GPS TYPE

Fig. 1 Error bars with the $95 \%$ CIs for the average positional errors. 
Measuring the Accuracy and Precision of the Garmin GPS Positioning in Forested Areas: A Case Study in Taxiarchis-Vrastama University Forest
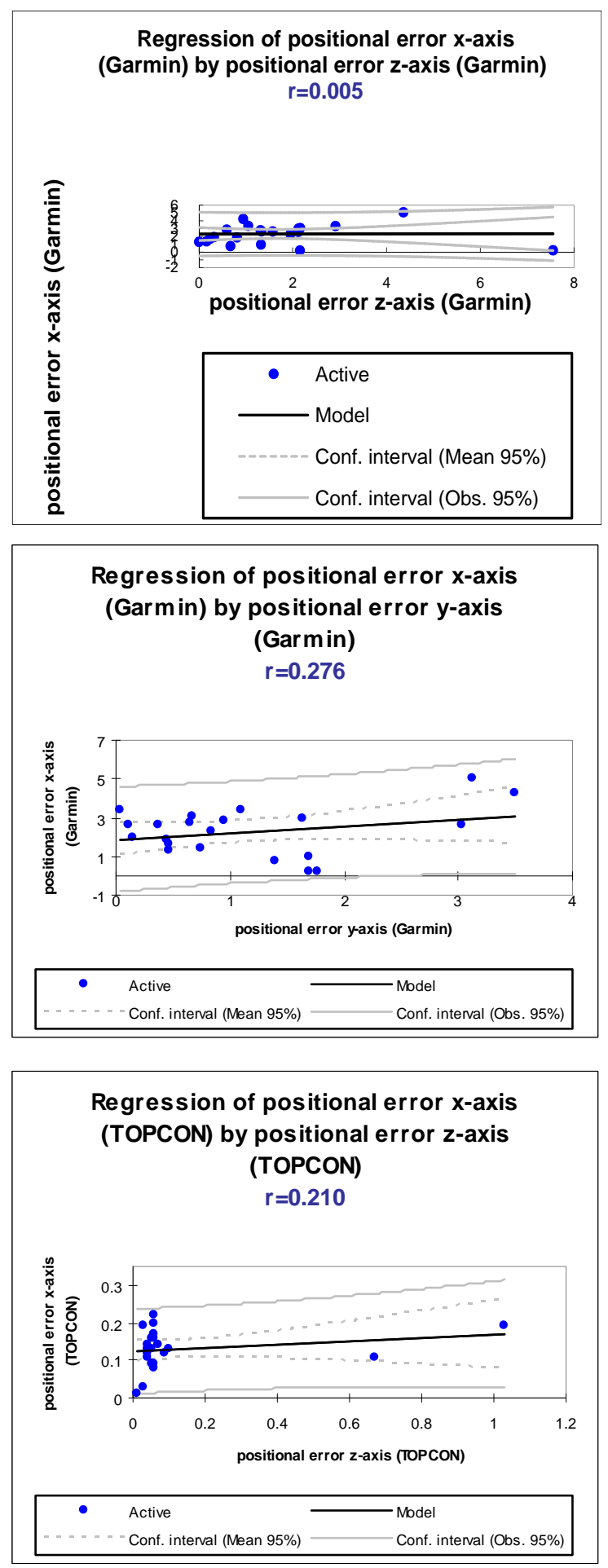
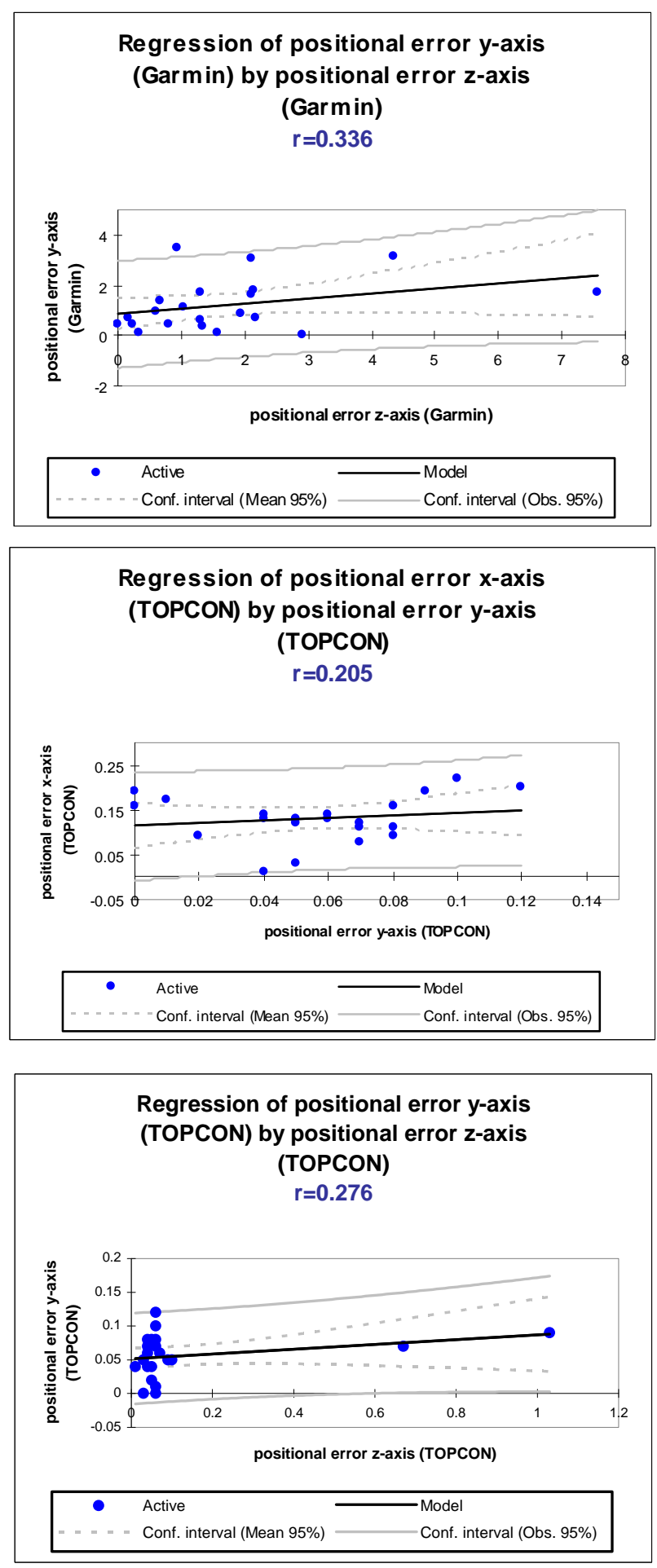

Fig. 2 Pearson correlation coefficients between the combinations of the positional errors of the axes.

measuring with this receiver they are going to get measurements that will fall 0.04887 meters within the true measurements $50 \%$ of the times.

As concerns the 3-dimensional accuracy of the two receivers under comparison (i.e. the combined horizontal and vertical accuracy), the spherical error probable is 2.014353 and 0.168538 for the Garmin and TOPCON, respectively, whereas the MRSE is 
Table 3 Measuring accuracy by receiver type.

\begin{tabular}{lll}
\hline \multirow{2}{*}{ Precision measures (m) } & \multicolumn{2}{c}{ Type of receiver } \\
\cline { 2 - 3 } & Garmin GPS & TOPCON GPS \\
\hline DRMS (2D) & 2.151787 & 0.061368 \\
2DRMS (2D) & 4.303575 & 0.122736 \\
CEP (2D) & 1.32361 & 0.04887 \\
R95 (2D) & 2.75311 & 0.10165 \\
MRSE (3D) & 2.738803 & 0.25404 \\
SEP (3D) & 2.014353 & 0.168538 \\
\hline
\end{tabular}

2.738803 and 0.25404 for the Garmin and TOPCON, respectively. In the sequel, data were analyzed in order to validate the effects of various factors on the obtained positional errors, such as the effect of positioning points, the GPS measurement system, and the direction (northing, easting and vertical). In doing this a GLM (generalized linear model) was fitted to the data, where the dependent variable was chosen to be the positional errors, whereas as the independent variables were chosen the above mentioned factors. Table 4 summarizes the obtained results concerning parameter estimates of the fitted model, along with the associated $p$-values.

The above fitted model explained $46.5 \%$ of the variation. As it follows from Table 4, the type of GPS is a significant factor for the positional error, at a $1 \%$ level of significance (beta $=1.62, p$-value $<0.001$ ). Indeed, as suggested by the model, the probabilities of higher positional error are increasing by a factor of 1.62 in case of using the Garmin GPS, when compared with the other GPS receiver.
Accordingly, positional error among the GPS positioning points differed statistically significantly (beta $=0.008, p$-value $=0.014<0.05)$. Finally, the positional error is more apparent in the $\mathrm{x}$-axis and $\mathrm{z}$-axis positioning, however this is not statistically confirmed by the significances of the associated factor. The final GLM regression model acquired from the fit is given by the following equation (Eq. (13)):

$$
\begin{aligned}
& \mathrm{D}_{\mathrm{i}}=1.62 \times(\text { GPS } \\
& (\text { TYPE })_{\mathrm{i}}+0.008 \times \\
& (\text { MEASUREMENT } \\
& \text { POINT })_{\mathrm{i}}+\mathrm{e}_{\mathrm{i}}
\end{aligned}
$$
where:

$D_{i}$ - the positional error $(\mathrm{m})$,

GPS $=0$ if GPS receiver is TOPCON, and GPS $=1$ if GPS receiver is Garmin;

$e_{i}$ stands for the error not explained by the model.

One of the most common factors affecting GPS accuracy is the occupation time required to achieve the claimed accuracy (see e.g. Refs. [13, 14]). Most systems are only able to achieve a considerable accuracy and precision after a stationary occupation of at least several minutes. Also, the geometric

\begin{tabular}{|c|c|c|c|}
\hline Parameter & Beta coefficient & $p$-value & $95 \%$ confidence interval \\
\hline intercept & -0.098 & 0.602 & $(-0.47-0.274)$ \\
\hline \multicolumn{4}{|c|}{ GPS Type (ref.: TOPCON) } \\
\hline Garmin GPS & 1.62 & $<0.001$ & $(1.283-1.956)$ \\
\hline \multicolumn{4}{|c|}{ Direction (ref.: Vertical) } \\
\hline Northing & -0.292 & 0.163 & $(-0.704-0.120)$ \\
\hline Easting & 0.3 & 0.152 & $(-0.112-0.712)$ \\
\hline Measurement point & 0.008 & 0.014 & $(0.002-0.014)$ \\
\hline$R$ Square & 0.465 & & \\
\hline Adjusted $R$ Square & 0.447 & & \\
\hline $\mathrm{N}=21$ & & & \\
\hline
\end{tabular}

Table 4 Obtained results concerning parameter estimates of the fitted model, along with the associated $p$-values.

Dependent variable: positional error $\left(D_{i}\right)$. 
distribution of GPS satellites, which changes according to time, affects GPS positional errors [14], although there are also studies claiming the opposite [2]. The number of visible GPS satellites during the field test for the two GPS types used is shown in Table 5.

According to Table 5, this field test was done only when the number of available GPS satellites was equal to or more than 6 , whereas the maximum number of visible GPS satellites was 8 . To examine the possible effects of the number of visible satellites on the accuracy of the measurement, once again, a GLM to the data including this time in the dependent variables of the model the number of satellites was fitted. The specific variable was considered in the analysis to be a categorical variable with three levels (i.e. with each level indicating the number of satellites during the time of measurement taken). The results of the analysis are summarized in Table 6.

Table 5 Number of visible GPS satellites during the field test.

\begin{tabular}{lll}
\hline GPS positioning point & \# of GPS satellites (Garmin) & \# of GPS satellites (TOPCON) \\
\hline 1 & 7 & 7 \\
2 & 7 & 7 \\
3 & 7 & 7 \\
4 & 7 & 7 \\
5 & 6 & 7 \\
6 & 6 & 7 \\
7 & 7 & 7 \\
8 & 8 & 7 \\
9 & 7 & 7 \\
10 & 7 & 7 \\
11 & 7 & 8 \\
12 & 7 & 8 \\
13 & 7 & 8 \\
14 & 7 & 8 \\
15 & 7 & 8 \\
16 & 8 & 8 \\
17 & 8 & 8 \\
18 & 7 & 7 \\
19 & 7 & 8 \\
20 & 7 & 7 \\
21 & 7 & 7 \\
\hline
\end{tabular}

Table 6 Obtained results concerning parameter estimates of the fitted model, including this time in the dependent variables of the model, the number of satellites.

\begin{tabular}{|c|c|c|c|}
\hline Parameter & Beta coefficient & $p$-value & $95 \%$ confidence interval \\
\hline intercept & -0.241 & 0.273 & $(-0.675-0.193)$ \\
\hline \multicolumn{4}{|c|}{ GPS Type (ref.: TOPCON) } \\
\hline Garmin GPS & 1.493 & $<0.001$ & $(0.141-1.844)$ \\
\hline \multicolumn{4}{|c|}{ Direction (Ref.: Vertical) } \\
\hline Northing & -0.292 & 0.157 & $(-0.699-0.114)$ \\
\hline Easting & 0.300 & 0.146 & $(-0.106-0.706)$ \\
\hline Measurement point & 0.008 & 0.008 & $(0.002-0.014)$ \\
\hline \multicolumn{4}{|c|}{ \# of Satellites (ref.: 8 Satellites) } \\
\hline 6 Satellites & 1.023 & 0.021 & $(1.154-1.892)$ \\
\hline 7 Satellites & 0.207 & 0.301 & $(-0.187-0.602)$ \\
\hline$R$ Square & 0.488 & & \\
\hline Adjusted $R$ Square & 0.463 & & \\
\hline $\mathrm{N}=21$ & & & \\
\hline
\end{tabular}

Dependent variable: positional error $\left(D_{i}\right)$. 
First of all, it can be seen from the goodness-of-fit statistics of the model that the inclusion of the extra explanatory variable has increased the percentage of variance in the positional errors explained by the model $(R$-square $=0.488$, adjusted $R$-square $=0.463)$. Further, there were no significant changes in the signs and significances of the variables entered as explanatory in the previous GLM model. In addition, the satellite factor was found to be significant in explaining variation in the positional errors.

Indeed, the results of Table 6 show that as the number of available visible satellites increases during the time period of the tests, the positional error reduces significantly. Specifically, while the results do not verify the improvement between a number of 8 and 7 satellites (beta $=0.207, p$-value $=0.301>0.05$ ), accuracy when measured with 6 satellites decreases statistically significantly in comparison with the presence of 7 or 8 satellites, at a 5\% level of significance $($ beta $=1.023$, $\mathrm{p}$-value $=0.021<0.05$ ). The estimated GLM model is expressed as (Eq. (14)):

$$
\begin{gathered}
\mathrm{D}_{\mathrm{i}}=1.493 \times\left(\mathrm{GPS} \_ \text {TYPE }\right)_{\mathrm{i}}+0.008 \times \\
(\text { MEASUREMENT_POINT })_{\mathrm{i}}+1.023 \times \\
(\# \text { _ OF_ SATELLITES })+\mathrm{e}_{\mathrm{i}}
\end{gathered}
$$

where,

$\mathrm{D}_{\mathrm{i}}=$ the positional error $(\mathrm{m})$;

GPS $=0$ if GPS receiver is TOPCON, and GPS $=1$ if GPS receiver is Garmin;

SATELLITES $=0$ for 7 and 8 observable satellites, respectively, and SATELLITES $=6$ for 6 observable satellites.

As already noted, one may claim that accuracy is also determined by length of time spent at the measurement point, also known as occupation time. In the sequel, to test for this effect and attempt to measure its impact on the accuracy of the measurement, it used time periods measured in minutes that were spent for collection of the TOPCON measurements (a total of 65 measurement points, accompanied by the real measurements, for computing the positional errors), and investigate the variables' relations to the TOPCON positional errors. Time spend ranged from 1 to 4 minutes, with an average of 2.12 minutes $(\mathrm{SD}=0.646$ $\min )$.

For positions determined for 1 and 2 minutes of observation, the mean error was 0.862 and $1.768 \mathrm{~m}$ respectively, whereas for 3 and 4 min observation the mean positional errors were found to be substantially lower ( $0.065 \mathrm{~m}$ and $0.078 \mathrm{~m}$, respectively).

Also, PDOP values of the receiver were included as an independent variable to represent geometric satellite distribution. In the literature, it is found various studies examining the associations between PDOP and accuracy of the measurement, with the majority of them reporting findings of no association at all between the two variables, or at least non-linear association.

For example, Næsset and Jonmeister [10] claim that PDOP is not a good indicator for positional accuracy (see Ref. [9] or [2]), whereas Næsset and others [14] finds a non-linear statistically significant association (multiplicative model).

The PDOP variable ranged from 2.4 to 3.6 , with an average of $3.03(\mathrm{SD}=0.34)$. However, such observation period values were not available in the current study for the GPS receiver, Garmin, thus the specific analysis is restricted only on the other receiver.

To this end, first of all, the correlation coefficients between the time variable and the three types of positional errors were calculated. It was found that the time spent was mainly negatively correlated with the vertical positional errors-as expected, with $r=-0.223$ $(p$-value $=0.075)$. Correlation between the $\mathrm{y}$-axis positional errors and time spent was substantially lower $(r=-0.032, p$-value $=0.803)$, while correlation between the time spent and the $\mathrm{x}$-axis positional errors was $-0.051(p$-value $=0.685)$. This indicates that measurement time spent affects more strongly the vertical accuracy of positions, than the horizontal accuracy.

Accordingly, the correlations between PDOP and positional errors were calculated and found to be non-significant (specifically, correlation between 
Table 7 Obtained results concerning parameter estimates of the fitted GLM model, using the TOPCON positional errors as the dependent variable.

\begin{tabular}{llll}
\hline Parameter & Beta coefficient & $p$-value & $95 \%$ confidence interval \\
\hline intercept & -5.683 & 0.557 & $(-24.748-13.383)$ \\
\hline Direction (Ref.: Vertical) & & & \\
\hline Northing & 1.809 & 0.315 & $(-1.736-5.355)$ \\
Easting & 1.599 & 0.375 & $(-1.947-5.144)$ \\
Measurement point & 0.045 & 0.373 & $(-0.054-0.143)$ \\
\hline \# of Satellites (ref.: 9 Satellites) & & & \\
\hline 6 Satellites & 0.109 & 0.982 & $(-9.590-9.808)$ \\
7 Satellites & 1.956 & 0.680 & $(-7.370-11.281)$ \\
8 Satellites & 1.522 & 0.734 & $(-7.288-10.331)$ \\
\hline Time spend for measurement (in min) (ref.: 4 min) & & \\
\hline 1 & -0.055 & 0.992 & $(-10.441-10.332)$ \\
2 & 0.910 & 0.842 & $(-8.072-9.892)$ \\
3 & -0.318 & 0.945 & $(-9.403-8.766)$ \\
PDOP & 0.764 & 0.776 & $(-4.535-6.064)$ \\
\hline$R$ Square & 0.017 & & \\
Adjusted $R$ Square & 0.036 & & \\
N =65 & & & \\
\hline Dep & & & \\
\hline
\end{tabular}

Dependent variable: positional error $\left(D_{i}\right)$.

$\mathrm{x}$-axis errors and PDOP was $0.188, p$-value $=0.133$, correlation between $\mathrm{y}$-axis errors and PDOP was $r=$ -0.149, $p$-value $=0.235$ and finally correlation for z-axis errors and PDOP $r=0.105, p$-value $=0.404$ ), indicating thus that there is no linear association between PDOP and errors.

In the sequel, a GLM model using the TOPCON positional errors as the dependent variable was fitted, whereas as predictors selected the direction, the positional points, number of satellites and in addition the time spent for measurement (measured in minutes). The results of the regression fit are shown in Table 7.

As we see, from the $p$-values obtained from the fit of the previous GLM regression model, none of the five predictors are significant factors in predicting the positional errors, for the TOPCON receiver. This in one sense is indicative of the robustness of positional errors obtained by the TOPCON, which are found to be constantly small, regardless of possible effects of the various factors. The low predictability of the fitted model is easily verified by the extremely small $R$-square value.

However, since that no linear association that was detected from the fit of the above model, various non-linear relationships between predictors and the dependent variable were further examined. And it has found as partly found in other studies that errors $D_{i}$ and PDOP values are strongly correlated in a non-linear way. Specifically, when fitting a model of both linear and logarithmic term for the PDOP, it can obtain the following model (Eq. (15)):

$$
\begin{array}{r}
\mathrm{D}_{\mathrm{i}}=323.4+477.471 \times(\text { PDOP })_{\mathrm{i}}+474.398 \times \\
(\ln \mathrm{PDOP})_{\mathrm{i}}+\mathrm{e}_{\mathrm{i}}
\end{array}
$$

The fitted logarithmic curve provided highly significant parameter estimates (that rejected the null hypotheses of zero parameter estimates for both PDOP and $\ln P D O P$ at a $5 \%$ level of significance). The specific model indicates that the $D_{i}$ 's are associated with PDOP partly linearly and partly logarithmic.

\section{Conclusions}

The issue of GPS accuracy can be complex and an ideal description of GPS accuracy will have reference to several factors. In this study, authors have made an attempt to examine the performance of two different types of GPS receivers, one advanced and highly 
accurate and one simpler. The results of the analysis showed that there were significant differences between the receivers regarding accuracy and precision in measuring coordinates and that to obtain the smallest positional errors under very dense canopies, more advanced receivers should be used. Specifically, the results of the study revealed that the smallest error was obtained for positions that were computed with the more sophisticated and more precise GPS receiver, namely TOPCON GPS.

The differences were more apparent in the $\mathrm{x}$ - and $y$-axis measurement errors and lower in the vertical axis. The results of the study concerning positional errors found were more or less in accordance with previously conducted analyses. For instance, as concerns the recreational GPS Garmin, an average positional error of 1.724 meters $(\mathrm{SD}=1.401 \mathrm{~m})$ was found, whereas Næsset and Jonmeister [10] report for an analogous low-cost GPS receiver mean positional errors ranging between 0.49 and $3.60 \mathrm{~m}$ under forest canopy. Results of similar magnitude were also reported by Yoshimura and Hasegawa [4]. However, there are also studies found in the literature where positional errors using low-cost receivers are substantially higher [12].

Regression analysis was applied to the data to assess which and how various factors affected the GPS measurement errors. The study demonstrated that the most common factors that should be included in a complete description of accuracy include the number of available satellites during the positioning calculations with the GPS and the actual position of measurements collected, especially when using low cost GPS receivers. Time spent for measurements is found to have no statistically significant association with positional errors in the case of the highly accurate GPS receiver, while on the other hand PDOP exhibited non-linear association with measurement errors.

\section{References}

[1] Y. Tachiki, T. Yoshimura, H. Hasegawa, T. Mita, T. Sakai, G. Nakamura, Effects of polyline simplification of dynamic GPS data under forest canopy on area and perimeter estimations, Journal of Forest Research 10 (2005) 419-427.

[2] P. Sigrist, P. Coppin, M. Hermy, Impact of forest canopy on quality and accuracy of GPS measurements, International Journal of Remote Sensing 20 (18) (1999) 3595-3610.

[3] I. Sawaguchi, K. Nishida, M. Shishiuchi, S. Tatsukawa, Positioning precision and sampling number of DGPS under forest canopies, Journal of Forest Research 8 (2003) 133-137.

[4] T. Yoshimura, H. Hasegawa, Comparing the precision and accuracy of GPS positioning in forested areas, Journal of Forest Research 8 (2003) 147-152.

[5] P.H. Dana, Global positioning system (GPS) time dissemination for real-time applications, Real-Time Systems 12 (1999) 9-40.

[6] E.D. Kaplan, Understanding GPS Principles and Applications, Artech House Inc, Boston, 1996.

[7] D. Karsky, K. Chamberlain, S. Mancebo, D. Patterson, T. Jasumback, Comparison of GPS Receivers under a Forest Canopy with Selective Availability off, USDA Forest Service Project Report 7100, 2000.

[8] H. Hasegawa, T. Yoshimura, Application of dual-frequency GPS receivers for static surveying under tree canopies, Journal of Forest Research 8 (2003) 103-110.

[9] E. Næsset, Effects of differential single- and dual-frequency GPS and GLONASS observations on point accuracy under forest canopies, Photogrammetric Engineering \& Remote Sensing 67 (2001) 1021-1026.

[10] E. Næsset, T. Jonmeister, Assessing point accuracy of DGPS under forest canopy before data aquisition, in the field and after postprocessing, Scandinavian Journal of Forest Research 17 (2002) 351-358.

[11] K. Pearson, Mathematical contributions to the theory of evolution, III. Regression, heredity and panmixia, Philosophical Transactions of the Royal Society of London 187 (1896) 253-318.

[12] J.R. Rodriguez-Pérez, M.F. Alvarez, E. Sanz, A. Gavela, Comparison of GPS receiver accuracy and precision in forest environments, practical recommendations regarding methods and receiver selection, in: Proceedings XXIII FIG Congress, Munich, Germany, Oct. 8-13, 2006.

[13] E. Næsset, Point accuracy of combined pseudorange and carrier phase differential GPS under forest canopy, Canadian Journal of Forest Research 29 (5) (1999) 547-553.

[14] E. Næsset, T. Bjerke, O. Ørstedal, L.H. Ryan, Contributions of differential GPS and glonass observations to point accuracy under forest canopies, Photogrammetric Engineering and Remote Sensing 66 (4) (2000) 403-407. 